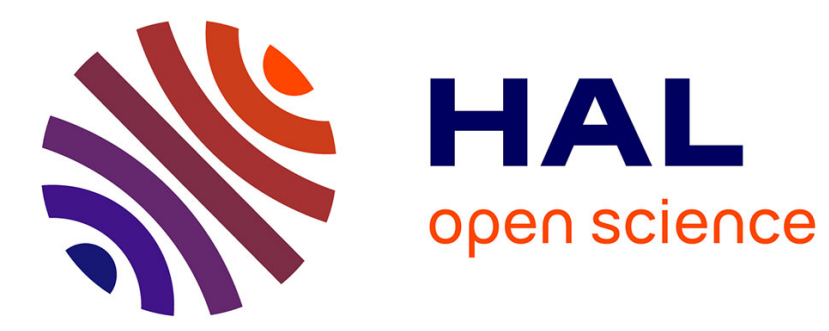

\title{
Passive compressive device in an MIMO configuration at millimeter waves
}

\author{
Antoine Jouadé, Olivier Lafond, Laurent Ferro-Famil, Mohamed Himdi, \\ Stéphane Méric
}

\section{- To cite this version:}

Antoine Jouadé, Olivier Lafond, Laurent Ferro-Famil, Mohamed Himdi, Stéphane Méric. Passive compressive device in an MIMO configuration at millimeter waves. IEEE Transactions on Antennas and Propagation, 2018, 66 (10), pp.5558-5568. 10.1109/TAP.2018.2862365 . hal-01904634

\section{HAL Id: hal-01904634 \\ https://hal-univ-rennes1.archives-ouvertes.fr/hal-01904634}

Submitted on 14 Dec 2018

HAL is a multi-disciplinary open access archive for the deposit and dissemination of scientific research documents, whether they are published or not. The documents may come from teaching and research institutions in France or abroad, or from public or private research centers.
L'archive ouverte pluridisciplinaire HAL, est destinée au dépôt et à la diffusion de documents scientifiques de niveau recherche, publiés ou non, émanant des établissements d'enseignement et de recherche français ou étrangers, des laboratoires publics ou privés. 


\title{
Passive Compressive Device in a MIMO Configuration at Millimeter-waves.
}

\author{
Antoine Jouadé, Olivier Lafond, Laurent Ferro-Famil, Mohamed Himdi and Stéphane Méric
}

\begin{abstract}
A passive compressive device (PCD), which works at millimeter-waves, is proposed in this study. In a passive manner, the device allows to compress the signals from multiple separate channels into a single or a reduced number of channels. This permits a simplification of the transmitter (Tx) or receiver ( $R x)$ chains for RADAR imaging applications. The PCD is a 3-Dimensional cavity that ensures each channel to be decorrelated with each other. If the transfer functions are known, the compressed signals are decompressed in post-processing. This article proposes a suitable method to estimate simultaneously the transfer functions of the PCD. This is validated by measurements in a Multiple-Input and Multiple-Output (MIMO) configuration to produce a complex Synthetic Aperture Radar (SAR) image. Measurements revealed that the point spread function generated by the MIMO configuration with the use of the PCD, leads to almost similar results, in terms of spatial resolution and sidelobe level ratio, as those of a Single-Input and Multiple-Output (SIMO) configuration (uniform amplitude), with fewer receiving elements.
\end{abstract}

Index Terms-millimeter-waves, radar imaging, passive compressive device, transfer function retrieval, SAR, wide-band, passive technique, MIMO.

\section{INTRODUCTION}

$\mathbf{I}$ $\mathrm{N}$ a RADAR imaging system, improving the spatial resolution is of main interest to better estimate scene reflectivity. Spatial resolution is inherently limited by the frequency band used and aperture dimensions. The aperture is sampled using a group of small radiating sources, whose amplitude and phase patterns may be determined so that they globally generate a beam that can be steered. By using Real Aperture Radar (RAR) imaging techniques, the beam is steered electronically (beamforming networks) or mechanically and the image reconstruction is performed sequentially. In Indirect imaging techniques, such as Array radar or Synthetic Aperture Radar (SAR), the image is reconstructed by means of signal processing techniques (digital beamforming). When the RADAR imaging is motionless, and if the area of interest is considered to be stationary in a given time interval, switch components may be used to select multiple receiving antennas sequentially through time [1]-[4]. In the non-stationary case, the signals that arrive at each receiving antenna have to be acquired simultaneously. This implies an entire Rx chain, from baseband to RF output, behind each source. For a large sampled aperture, the cost and the complexity is increased. A Multiple-Input Multiple Output (MIMO) array [5]-[8] is of considerable interest as

The authors are with the Institute of Electronics and Telecommunications of Rennes (IETR), France, e-mail: (antoine.jouade@univ-rennes1.fr).

Manuscript received April 19, 2005; revised August 26, 2015. it has the capability to generate a large virtual array from a reduced number of Tx and Rx chains. However, even with a reduced number of Tx and Rx chains, it is still complex and expensive to realize. If the backscattered signals that arrive upon the independent receiving antennas are compressed or coded in such a manner that the transfer functions are decorrelated/orthogonal from each other, signals from all receiving antennas may then be acquired with only one or few Rx chains. Subsequently, by decompressing the received signals, the contribution arrived at each receiving antenna can be extracted. It also works in the same manner for multiple transmitting antennas. The compression can be done in various active methods such as generating numerically multiple baseband waveforms [9], using a frequency division multiplexing [10], [11] or by employing space-time coding [12] to achieve diversity. The signals are usually coded in baseband before being up-converted around the carrier frequency.

A new method, which has recently emerged, uses the Passive Compressive Device (PCD). It takes advantage of frequency diversity inside a cavity, where the electromagnetic (EM) fields interfere destructively or constructively to create multiple modes, which vary with frequency. Two slightly different configurations can be distinguished. In the first one, the modes excite independent sources (slots, holes...) to produce distinct mode patterns as a function of frequency [13]-[17] or use a compressive reflector antenna that generates spatially coded patterns after reflecting the incident fields produced by a feeding array [18]. To reconstruct the image, a complete characterization of the aperture field distribution is necessary using near-field scanning measurements [19]. The measured fields are then propagated over the area of interest. In such a case, both the radiating elements and the cavity are used to reconstruct the image. The second configuration is to have multiple input ports and multiple output ports on the cavity's surface. Each port may be a coaxial connector or a waveguide port, that allows for a direct measurement of the complex transfer function along the frequency from one input to one output port by using a Vector Network Analyzer (VNA). Finally, the ports are connected to antennas whose properties (locations, types, directivities, polarizations...) may be modified as desired without changing the overall system configuration [20]-[23]. The latter configuration is the one used in this study as it can be adapted for use in various RADAR imaging configurations. A particular attention is given on the retrieval of the transfer functions and a method to estimate simultaneously the transfer functions under real 
conditions of use is proposed.

Hence, a $N \times M$ passive device allows for the compression, at the RF level, of $\mathrm{N}$ signals into $\mathrm{M}$ signals or $\mathrm{M}$ signals into $\mathrm{N}$ signals either for the reduction of the number of Tx chains in a MISO or MIMO configuration or for the reduction of the number of Rx chains in a SIMO or MIMO configuration. Furthermore, multiple PCDs may be used simultaneously. The correlation between the compressed signals is mainly dependent on the size and the shape of the device. Nonetheless, if it is used in transmission, the main drawback of using a PCD is that the limited transmitted power is shared among the output ports, implying a reduction of the range of detection. However, amplifiers may be used to counter the attenuations. Further, the transmitted powers delivered at the input ports are, in fact, not only shared among the output ports but also shared with the input ports. The power reflected back to the input ports has to be considered as a loss. Such a PCD has, by essence, low efficiency. However, these drawbacks have to be compared with the capability of the device to reduce the number of Tx and Rx chains, which sustains considerable interest, particularly at millimeter-waves because it permits having a reasonnable cavity size with significant compression. The ports facing the scene are named as output ports whereas those connected to the radio-frequency chains are named as input ports in this article. The $N \times M$ compressive device with $N$ inputs and $M$ outputs can be used among numerous of RADAR configurations. A few of them are listed below:

- MIMO RADAR either in transmission or reception or both simultaneously [24], to generate a uniform or a nonuniform array depending on the location of each port.

- Synthetic Aperture RADAR (SAR) [25], Multi-beam radar [26] or Multi-beam SAR configuration [27] if the antennas at each output port are looking at different angles.

- Moving Target Identification (MTI) in a SAR configuration [28] if the output ports are regularly placed in the along-track direction (Multi-channel SAR MTI by using either Displaced Phase center Antenna (DPCA), Along track interferometry (ATI) or Space time adaptive processing (STAP)).

- SAR Interferometry (In-SAR) [29], [30] for tomography purpose if the output ports are placed along the elevation direction.

- SAR Polarimetry (Pol-SAR) [31] if the output port polarizations are alternatively changed.

In Section II, details of the PCD and the associated antennas module are given. Section III shows two methods to measure the transfer functions by using (i) classic Port-to-Port measurements through a VNA and (ii) by estimating the transfer functions from measurements performed in a Compact Antenna Test Range (CATR) where the plane-wave assumption is considered. To validate both methods, the PCD is used in a MIMO configuration in Section IV to analyze the point spread function resolution from measurements and are compared to a Single-Input-Multiple-Output (SIMO) configuration with a synthetic aperture length of the same size as the virtual array length generated by the MIMO configuration.

\section{PASSIVE COMPRESSIVE DEVICE}

From [32], a cavity big enough, in terms of wavelength, excited over a wide frequency bandwidth exhibits spatial and spectral statistical behaviors very close to the random theory, due to the large number of modes that are present inside the cavity. For an asymmetric cavity, the fluctuation of the combination of all the modes (i.e. mode diversity) along the frequency increases as the number of modes increases (larger cavity dimension). By minimizing the number of outputs, the scattering inside the cavity increases, which in turn, leads to increased mode diversity. If the transfer functions are known, the PCD permits to modulate, by different series of orthogonal waveforms, the signal at each output, so that these signals can be separated in post-processing. Then, the transfer functions, which correspond to each channel, satisfy the orthogonality requirement.

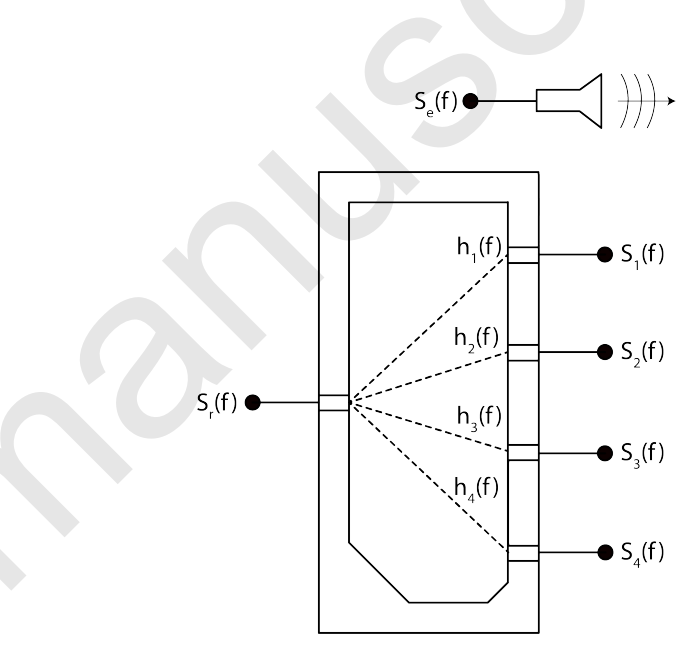

Fig. 1. Cutting view drawing of the $1 \times 4$ passive device with the associated transfer functions $h_{m}(f)$ for $m=1, \ldots, 4$.

To maintain the power at an acceptable level, at the outputs of the device, we choose to use a $1 \times 4$ PCD with output ports that perform a uniform linear array (see Fig. 1).

In a 3-Dimensional spatial space, a transmitting antenna has a phase center located at a fixed position $\left(x_{t}, y_{t}, z_{t}\right)$. The PCD is used as the receiving part with $M=4$ linearly spaced receiving antennas. The phase centers are aligned with the $\mathrm{x}$ axis and with an element spacing of $\Delta_{x}$. Their locations are $\left(x_{a}, y_{a}, z_{a}\right)$ with $\left\{x_{a}(m)=-\Delta_{x} \cdot(M-1) / 2+(m-1)\right.$. $\left.\Delta_{x}\right\}_{m=1}^{M} . N_{s}$ point-like scatterers identified by their Cartesian coordinates $\left(x_{i}, y_{i}, z_{i}\right)$ are considered. The index $i$ yields for the $i^{t h}$ point scatterer $P_{i}$. The equivalent distance for the wave that propagates from the transmitter to the point scatterer $P_{i}$ to the $m^{\text {th }}$ receiving antenna of the PCD is given by:

$$
\begin{aligned}
d_{i}(m) & =\left(d T x_{i}+d R x_{i}(m)\right) / 2 \\
& =\left(\sqrt{\left(x_{t}-x_{i}\right)^{2}+\left(y_{t}-y_{i}\right)^{2}+\left(z_{t}-z_{i}\right)^{2}}\right. \\
& \left.+\sqrt{\left(x_{a}(m)-x_{i}\right)^{2}+\left(y_{a}-y_{i}\right)^{2}+\left(z_{a}-z_{i}\right)^{2}}\right) / 2
\end{aligned}
$$

A RF stimulus signal $\left(S_{e}(f)\right)$ is transmitted over a frequency bandwidth $B_{f}$. Prior to being sent through the medium, the signal is transposed around the carrier frequency $f_{c}$. The backscattered received signals, at the output of the PCD, are 
modeled as a sum of transmitted signals that are weighted and delayed. The complex weighting $s_{i}$ represents all the attenuations that occur during the round-trip propagation and the reflectivity of the $i^{t h}$ point scatterer $P_{i}$. The delays arise from the round-trip distances determined in (1). Once the signals have reached the receiving antennas (output ports), they are transmitted inside the PCD (i.e. the cavity) to be received by the input port. The propagation inside the PCD from the $m^{\text {th }}$ port to the input port corresponds to a multiplication, in the frequency domain, between the receiving signals $S_{m}(f)$ and the $m^{t h}$ transfer function $\dot{h}_{m}(f)$.

$\dot{h}_{m}(f)$ is the transfer function along the entire frequency spectrum. Furthermore, the signal is affected by $n(f) \sim \mathcal{N}_{\mathbb{C}}\left(0, \sigma^{2}\right)$, a Gaussian white noise with zero mean and variance $\sigma^{2}$.

The received signals at the input port of the PCD can be modeled as:

$$
\begin{aligned}
S_{r}(f) & =\sum_{m=1}^{M} \dot{h}_{m}(f) \cdot S_{m}(f)+n(f) \\
& =\sum_{m=1}^{M} \dot{h}_{m}(f) \cdot \sum_{i=1}^{N_{s}} s_{i} S_{e}(f) e^{-j 4 \pi\left(f+f_{c}\right) d_{i}(m) / c}+n(f)
\end{aligned}
$$

with $c$ being the speed of light. A transmitted signal $S_{e}(f)$ is considered with a flat unitary spectrum over a limited frequency bandwidth $B_{f}$. By introducing $h_{m}(f)$ as the transfer function along the limited frequency bandwidth, the following is obtained:

$$
h_{m}(f)=S_{e}(f) \dot{h}_{m}(f)=\operatorname{rect}\left(\frac{f-f_{c}}{B_{f}}\right) \dot{h}_{m}(f)
$$

Introducing (3) into (2) gives:

$$
S_{r}(f)=\sum_{m=1}^{M} h_{m}(f) \cdot \sum_{i=1}^{N_{s}} s_{i} e^{-j 4 \pi\left(f+f_{c}\right) d_{i}(m) / c}+n(f)
$$

In the presence of additive noise, the matched filter is the optimal filter for maximizing the signal-to-noise ratio [33]. Then, on the received signal, four different matched filters are applied to extract the received signals that have been acquired by the four output ports. If the transfer functions $h_{m}(f)$ are known, the received signals that come from the $m^{\text {th }}$ output port are extracted by the matched filtering as shown in (5).

$$
\begin{aligned}
S_{m}(f) & =S_{r}(f) h_{m}^{*}(f) \\
& =h_{m}(f) h_{m}^{*}(f) \cdot \sum_{i=1}^{N_{s}} s_{i} e^{-j 4 \pi\left(f+f_{c}\right) d_{i}(m) / c} \\
& +\sum_{\substack{l \neq m \\
l=1}}^{M} h_{l}(f) h_{m}^{*}(f) \cdot \sum_{i=1}^{N_{s}} s_{i} e^{-j 4 \pi\left(f+f_{c}\right) d_{i}(l) / c} \\
& +n_{f i}(f)
\end{aligned}
$$

The first term is the useful received signal and the second term is the cross-correlation with the other ports. $n_{f i}(f)=n(f) h_{m}^{*}(f)$ being the filtered white noise by means of the matched filtering. In the ideal case, when the transfer functions are uncorrelated, the second term vanishes.

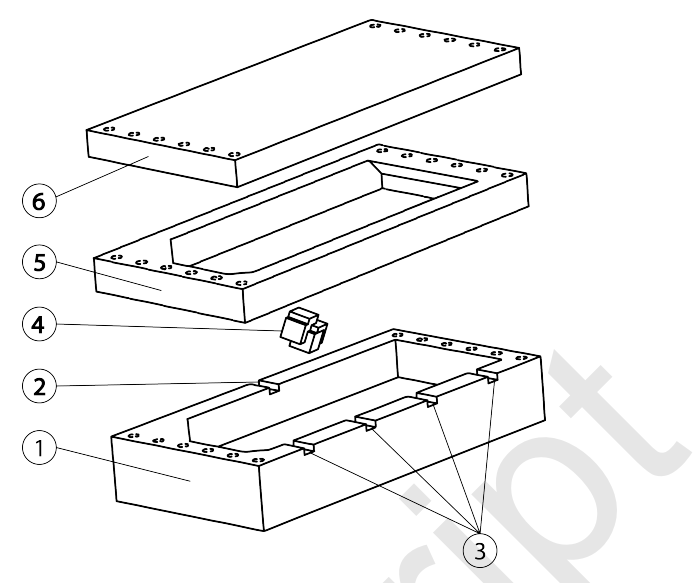

Fig. 2. Exploded view drawing of the passive device with 1) the lower part with 2) the input and 3) the output ports. 4) the diffractive element at the center of the cavity and 5/6) the upper parts closing the cavity.

The key factor is to manufacture a PCD whose transfer functions are relatively well decorrelated so that they can be discriminated. The main parameter is the volume of the cavity, which steers the number of modes it can generate, and fixes the level of randomness of its transfer function. Some of the vertical and horizontal corners are bent in such a manner so as to avoid any symmetry inside the cavity. The optimized PCD (see Fig. 2) has an overall dimension of $115 \mathrm{~mm}$ length by $55 \mathrm{~mm}$ width by $35 \mathrm{~mm}$ height $(23 \lambda \cdot 11 \lambda \cdot 7 \lambda$ at $60 \mathrm{GHz}) . \lambda=c /\left(f+f_{c}\right)$ is the wavelength. The walls, made of aluminum, are $7.5 \mathrm{~mm}$ thick. Furthermore, a diffractive element is located at the center of the cavity. It is composed of three $0.75 \times 0.75 \times 0.75 \mathrm{~mm}^{3}$ cubes that are overlapped and slightly displaced from one another along the three dimensions. The element provides sharp edges that allow the incoming wave to be diffracted. It is used to decrease the level of the reflection coefficient at the input port, as the wave is diffracted in all directions. Furthermore, it is used to improve the spectral statistical behaviors of the cavity by allowing a better decorrelation between the transfer functions. One of the corners of the diffractive element is welded to the bottom of the cavity. The cavity is excited by a standard Vband waveguide (WR-15) for an operating frequency band of 50-66 GHz.

There are four output waveguide ports placed to have a uniform linear array with an element spacing of $25 \mathrm{~mm}$ $(5 \lambda$ at $60 \mathrm{GHz}$ ). A non-linear array may be preferable to decrease the correlation between each port. Nevertheless, the linear array has been chosen in a MIMO configuration, for creating a fully populated virtual array. The output ports have the same vertical polarization as the input port polarization. The element spacing and the dimension of the cavity have been chosen so that a round flange waveguide of diameter $19 \mathrm{~mm}$ can be connected at each output. It is used to measure the transfer function of each channel. The realized PCD is shown in Fig. 3a. 13 screws are used to attach the different parts and to minimize the presence of air gaps. No electromagnetic simulation software has been used, since deterministic responses may reveal useless; small irregularities 
or modifications between the simulated cavity and the realized one may involve, due to the chaotic nature of the device, extreme changes in the actual random values of the transfer functions, while keeping second-order statistical indicators, correlation functions, globally unchanged.

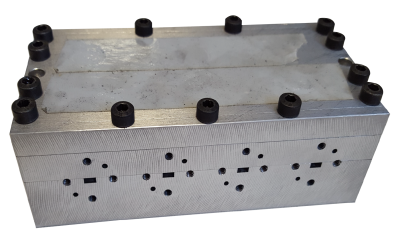

(a)

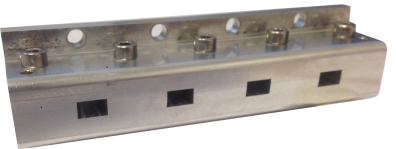

(b)
Fig. 3. Photography of (a) the output face of the realized PCD, which works at millimeter-waves, and (b) the front-end horn antenna module.

A front-end horn antenna module (see Figs. 3b and 4), made of aluminum, is attached at the output of the PCD to slightly focus the beams in a limited angular sector. Four similar horn antennas are used with a $8 \times 5 \mathrm{~mm}^{2}$ aperture size and with a horn length of $10 \mathrm{~mm}$. The reflection coefficient is lower than $15 \mathrm{~dB}$ and the coupling between each port is lower than $-55 \mathrm{~dB}$ over the frequency range. Figs. $4 b$ and $4 c$ show the measured radiation patterns of the first horn fixed to the first port of the PCD along the E-plane and H-plane, respectively. The gain of the antenna varies from 11 to $15 \mathrm{dBi}$ in the frequency range (50 to $66 \mathrm{GHz}$ ). Along the E-plane, the Half-Power Beam Width (HPBW) varies from $60^{\circ}$ to $50^{\circ}$ over the frequency range. Along the H-plane $(G(\theta, f))$, the HPBW varies from $48^{\circ}$ to $38^{\circ}$ over the frequency range. The side-lobe level (SLL) is lower than $-40 \mathrm{~dB}$ for the $\mathrm{H}$-plane and lower than $-25 \mathrm{~dB}$ for the E-plane.

\section{TRANSFER FUNCTIONS RETRIEVAL}

\section{A. Direct Port-to-Port measurement using a VNA}

The knowledge of the transfer functions is required to decorrelate the received signals. For a low number of ports, it is possible to measure individually each transfer function using a 2-port VNA. A full waveguide-port calibration is performed. The first port of the VNA is connected to the input port of the device, whereas the second port of the VNA is connected to the $m^{t h}$ output port of the device and loads are attached to the other ports. The measurement of the complex transmission coefficient at each frequency provides the transfer function of the $m^{t h}$ channel. The transfer functions are measured from 50 to $66 \mathrm{GHz}$ with a frequency step of 80 $\mathrm{MHz}$, which corresponds to 2001 frequency bins. The wide frequency bandwidth is required to enable a full decorrelation capability to the PCD. The $80 \mathrm{MHz}$ frequency step $\left(\delta_{f}\right)$ allows distinct frequency measurements for imaging. The PCD has a quality factor $Q=f_{c} / \delta_{f}$ of about 725 . The measured transfer functions within the frequency band are shown in Fig. 5. The spectral statistical behaviors of the transfer functions are very close to a random process.

Figure 6 shows the autocorrelation of each measured transfer function $\left(\mathbf{r}_{\mathbf{i j}}(\tau)\right.$ for $\left.i=j\right)$, along the diagonal; above and below the diagonal, the mutual cross-correlation between each port are shown. Over the cross-correlation results, the

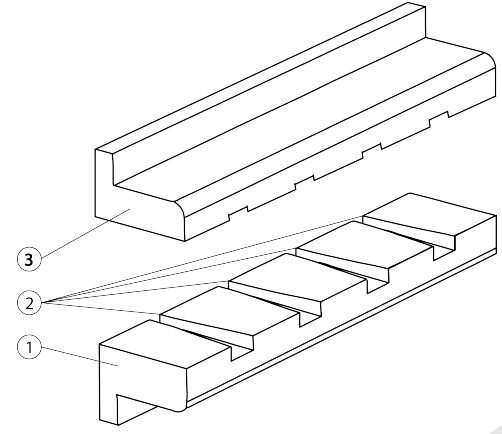

(a)

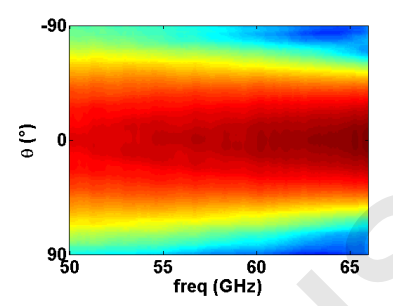

(b)

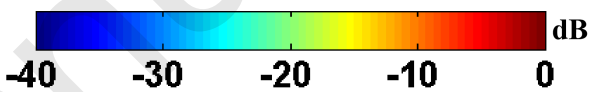

Fig. 4. Details of the antenna module with four similar horn antennas with a $8 \times 5 \mathrm{~mm}^{2}$ aperture size (a) Exploded view drawing of the horn antenna module with 1) the lower part, 2) the input ports attached to the output ports of the PCD, (3) the upper part. The measured radiation pattern of the first port along (b) the E-plane Co-polar and (c) H-plane Co-polar $(G(\theta, f))$.

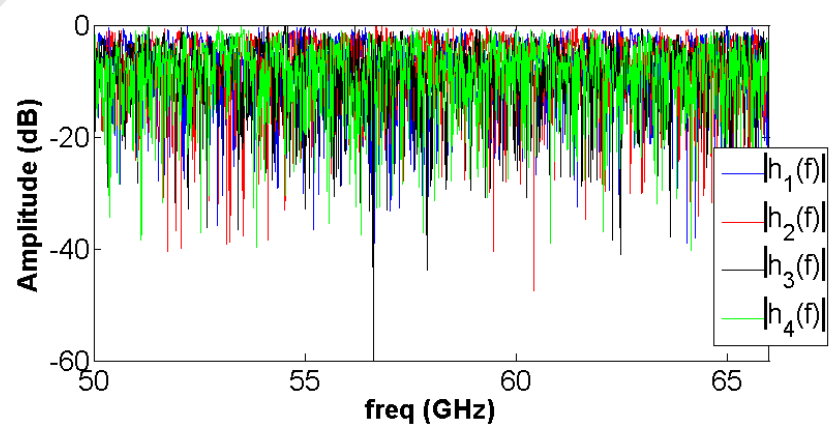

Fig. 5. Absolute value $[\mathrm{dB}]$ of the measured transfer functions by means of a VNA along the frequency band.

highest measured peak reaches $-15.5 \mathrm{~dB}$ as compared to the measured autocorrelation functions. The peak side lobe ratio (PSLR) [34] of the autocorrelation functions is lower than -20 $\mathrm{dB}$ from the main lobe peaks and with an integrated side lobe ratio (ISLR) [34] lower than $-14.7 \mathrm{~dB}$. The power efficiency $\eta$ of the PCD is shown in Fig. 7. The power efficiency $\left(\eta(f)=10 \log _{10}\left(\sum_{m=1}^{4}\left|h_{m}(f)\right|^{2}\right)\right)$ has a mean value of $5.17 \mathrm{~dB}$, also named as the average radiation efficiency and is controlled by both the reflection coefficient at the input port and the losses that occur inside the cavity because of multiple reflections on aluminum walls.

However, the PCD may be used with specific front-end without access to the output ports. In such a case, measure- 

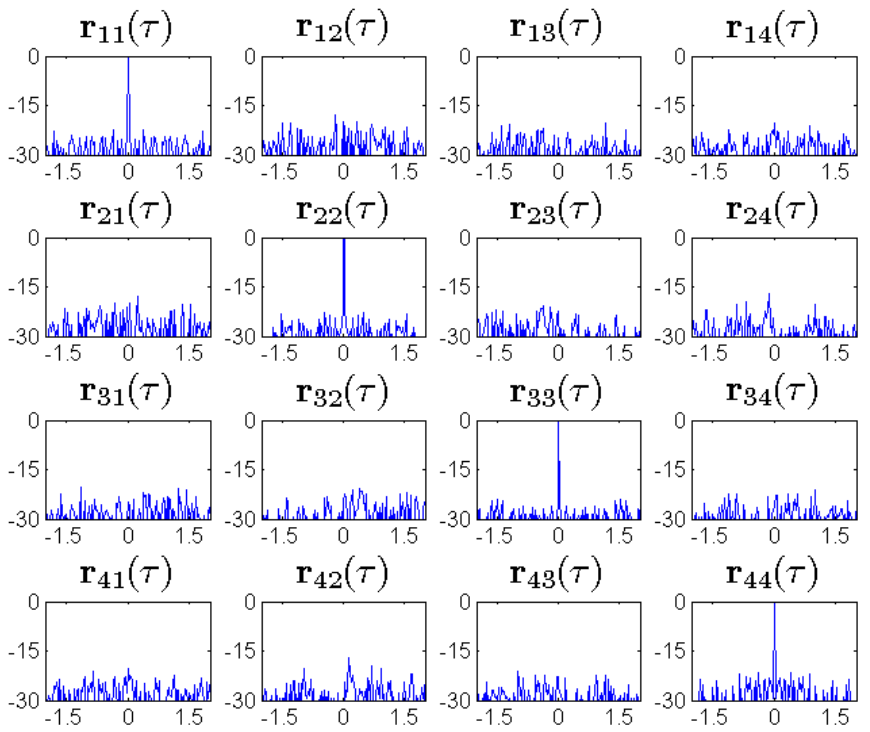

Fig. 6. $\mathbf{r}_{\mathbf{i j}}(\tau)=D F T^{-1}\left[h_{i}(f) h_{j}(f)^{*}\right]$, the time-domain correlation results between the measured transfer functions (VNA measurements), with the time domain $\tau$ [ns] along the horizontal axis and the normalized amplitude [dB] along the vertical axis.

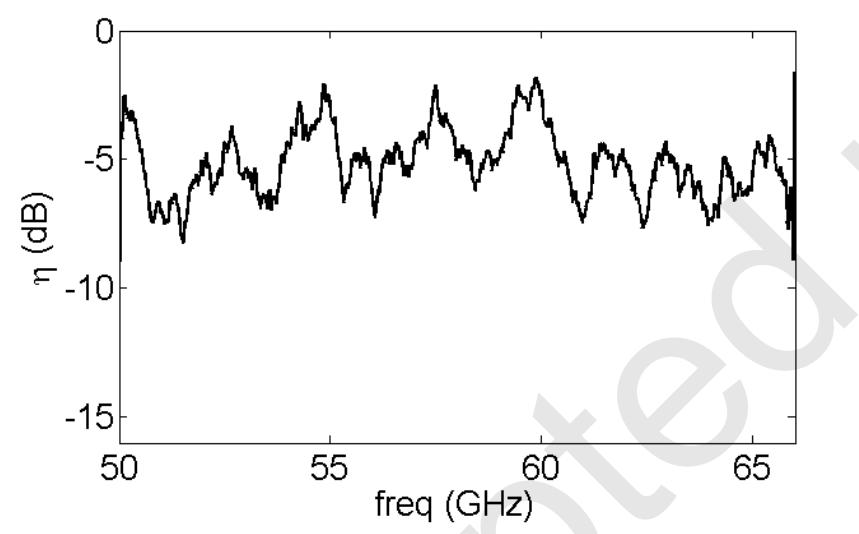

Fig. 7. Measured power efficiency of the passive device, which corresponds to the outgoing powers from the four ports versus the incoming power, along the frequency.

ments of the transfer functions are not possible. In addition, when a larger number of ports are needed, measuring individually each transfer function may become complex. Furthermore, the loads used being not perfect, a portion of the signals that arrive at the loads is reflected back inside the cavity. Such behavior may cause errors when measuring the transfer functions.

One solution may be to perform near-field scanning measurements to retrieve the transfer functions; however, the probe and the scanning system used may interact with the PCD, leading to measurement errors. The next section presents a procedure that allows for the estimation of the transfer functions of a $N \cdot M$ PCD. The procedure is tested on the fabricated $1 \times 4$ PCD.

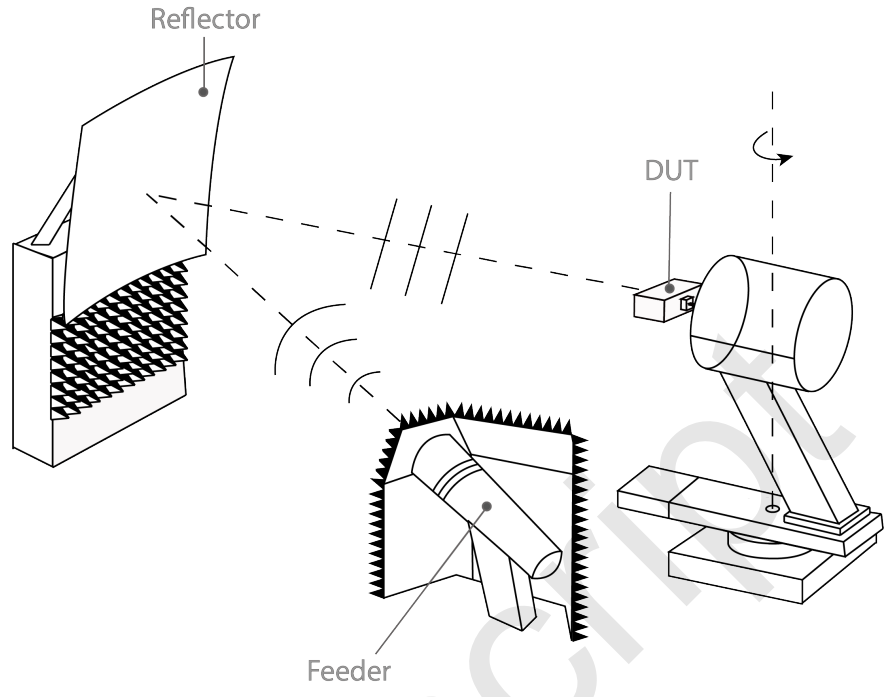

Fig. 8. Setup measurement by using a compact antenna test range.

\section{B. Plane-wave estimation using Compact Antenna Test Range measurements}

The measurements are performed in the Compact Antenna Test Range (CATR) IETR facility [35]. The RF measurement system uses a classic architecture with a VNA and external VDI (Virginia Diodes, Inc) TxRef and Rx frequency extenders. It is dedicated to measure high gain antennas at a short distance, through the capability of a AL-25202 $1.2 \mathrm{~m} \times 1.2 \mathrm{~m}$ rolled edge corner-fed compact range reflector to create a quiet zone at a fixed range of diameter $600 \mathrm{~mm}$ with a depth cylinder of $600 \mathrm{~mm}$ with sufficient accuracy (amplitude ripples lower than $0.3 \mathrm{~dB}$ and phase ripples lower than $10^{\circ}$ ). Hence, inside the quiet zone, the amplitude of the electric field is considered as uniform and the plane-wave assumption can be used. Fig. 8 shows the measurement setup.

An optimized horn antenna (feeder) illuminates the reflector which allows for transformation of a spherical wavefront onto a plane wavefront within the quiet zone. Inside the quiet zone is placed the Device Under Test (DUT), attached to a receiver, which can be rotated along the azimuth direction. The received signal at the input port of the PCD forms a linear combination between the received signals at each output port and the associated transfer functions. When rotating the PCD, the transfer functions are not modified and only the phase along the output ports varies in a known manner. Hence, the rotation along the azimuth direction gives the diversity needed to extract the transfer functions of the PCD.

Since the location of each output port is a known parameter, the phase shift between each port, based on the frequency and the angle of incidence of the plane wave, is used to design a steering vector according to the plane-wave assumption.

The round-trip carrier wavenumber is described as $k_{c}=$ $4 \pi f_{c} / c$, whereas the baseband wavenumber is $k=4 \pi f / c$. By inserting the azimutal diversity $\theta$ in (4), with $N_{s}=1$, it yields: 

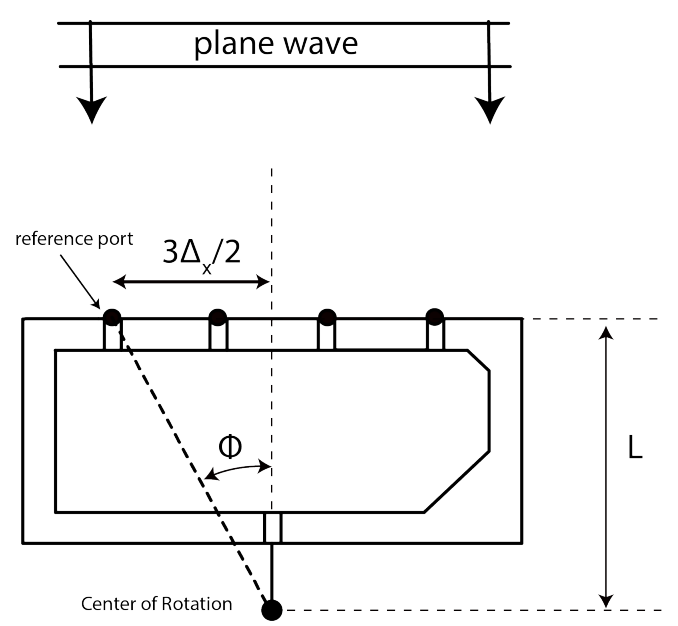

(a)

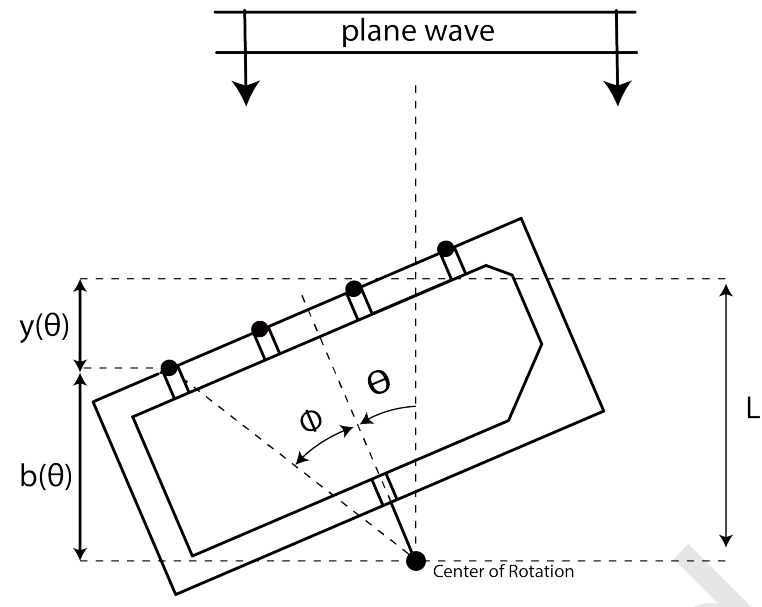

(b)

Fig. 9. Sketch of the phase variation that occurs when the DUT is looking (a) broadside and (b) at squint angle.

$$
S_{r}(\theta, k)=\sum_{m=1}^{M} h_{m}(k) \cdot s_{1} G(\theta, k) e^{-j \varphi_{m}(\theta, k)}+n(\theta, k)
$$

with $G(\theta, k)$, the Co-polar H-plane radiation pattern of each antenna and $\varphi_{m}(\theta, k)=\left(k+k_{c}\right) d_{1}(\theta, m) / 2$ the phase variation at the $m^{\text {th }}$ output port along the spatial frequency and azimuthal diversities. $s_{1}$ is the complex weighting coefficient, which represents the attenuations that occur during the roundtrip propagation from the unique wave that arrives on the DUT. $d_{1}(\theta, m)$ is the distance between the transmitting antenna, the reflector, and the $m^{t h}$ port of the PCD when it is rotated at an angle $\theta$ from broadside. Using the plane-wave assumption that is valid in the quiet zone, the phase variation can be approximated according to the first order Taylor expansion of the distance. By taking as a phase reference the first port of the PCD, it yields:

$$
\begin{aligned}
\Psi_{m}(\theta, k) & =\varphi_{m}(\theta, k)-\varphi_{1}(\theta, k) \\
& =\left(k+k_{c}\right)\left(d_{1}(\theta, m)-d_{1}(\theta, 1)\right) / 2 \\
& \approx-\left(k+k_{c}\right)(m-1) \Delta_{x} \sin (\theta) / 2
\end{aligned}
$$
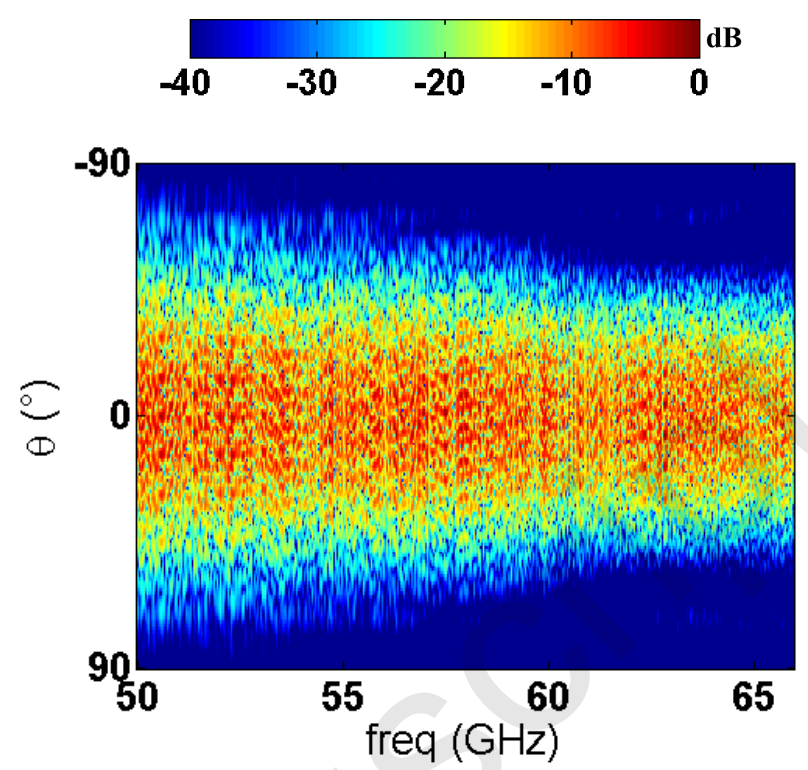

(a)

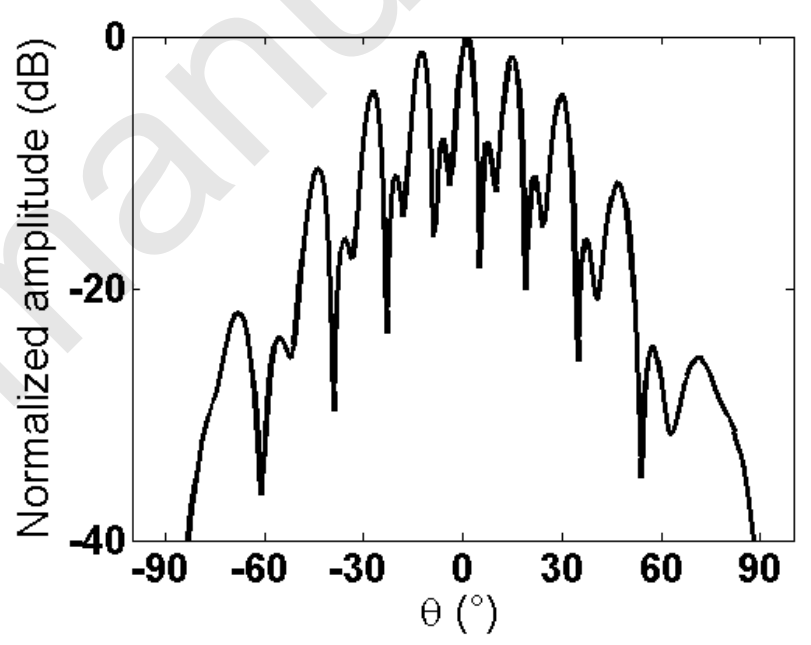

(b)

Fig. 10. Normalized measured data along (a) $\left|S_{r}(\theta, f)\right|[\mathrm{dB}]$ (b) $\left|S_{r}\left(\theta, f_{0}\right)\right|$ $[\mathrm{GHz}]$ with $f_{0}=f+f_{c}=50 \mathrm{GHz}$.

A finite range of azimuth angles is used, with $\theta \in$ $\left[\theta_{\min }, \theta_{\max }\right]$ for $N_{\theta}$ scanning angles. The difference in phase (phase shift), generated by the plane-wave that arrives at an angle $\theta$ from broadside between two ports, is $\Psi=-(k+$ $\left.k_{c}\right) \Delta_{x} \sin \theta / 2$ with $\Delta_{x}$ the distance between the ports. The steering vector, which contains the phase shifts, is defined as $\mathbf{a}(\Psi)=\left[1, e^{-j \Psi}, \ldots, e^{-j(M-1) \Psi}\right]$. It yields the steering vector matrix by considering all the phase shifts at each port for all scanning angles such as $\mathbf{A}=\left[\mathbf{a}\left(\Psi_{1}\right), \ldots, \mathbf{a}\left(\Psi_{N_{\theta}}\right)\right]^{T}$. The receiving signal at the input port of the PCD can be modeled as follows:

$$
\hat{\mathbf{S}}_{r}(\theta, k)=\mathbf{A} \hat{\mathbf{h}}(k)
$$

with $\hat{\mathbf{h}}(k)=\left[\hat{h}_{1}(k), \ldots, \hat{h}_{M}(k)\right]^{T}=\left[\alpha_{1}(k)+\right.$ $\left.j \beta_{1}(k), \ldots, \alpha_{M}(k)+j \beta_{M}(k)\right]^{T}$ the estimated transfer functions vector at the wavenumber $k+k_{c}$ with $\alpha_{i}$ and $\beta_{i}$, respectively, the real part and the imaginary part of the $i^{t h}$ transfer function, 
which has to be estimated. This model is valid if the location of the first port (reference) is unchanged during the rotation, which is not the case in this study. This effect, indicated in Fig. 9, has to be included in (8) to compensate for the phase variation when the receiving part is rotating. The phase shift, linked to the distance variation of the first port due to the rotation, is defined as $\delta(\theta, k)=\exp \left(j\left(k+k_{c}\right) y(\theta) / 2\right)$ with $y(\theta)=L-b(\theta)=L-\sqrt{\left(3 \Delta_{x} / 2\right)^{2}+L^{2}} \cdot \sin (\pi / 2-\phi-\theta)$ and with $\phi=\operatorname{atan}\left(\frac{3 \Delta_{x}}{2 L}\right)$. Noting $(\bullet)$ the element-wise product, it yields:

$$
\hat{\mathbf{S}}_{r}(\theta, k)=\mathbf{A} \hat{\mathbf{h}}(k) \bullet \delta(\theta, k)
$$

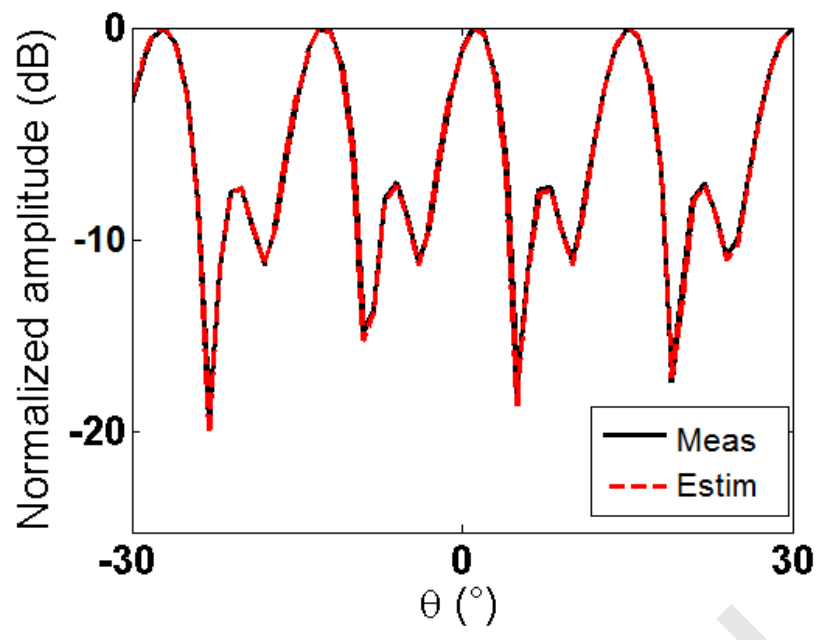

(a)

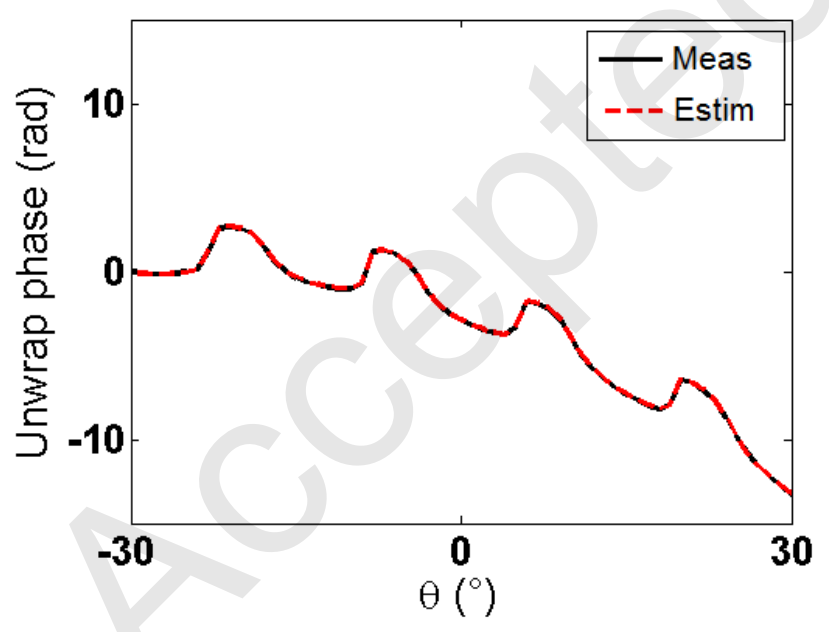

(b)

Fig. 11. Measured data at $f_{0}=50 \mathrm{GHz}$ (a) $\left|S_{r}\left(\theta, f_{0}\right)\right|[\mathrm{dB}]$ and (b) $\arg \left(S_{r}\left(\theta, f_{0}\right)\right)$ [rad]. The black line corresponds to the normalized measured data $\left(\mathbf{S}_{r}(\theta, f)\right)$ and the dashed red line corresponds to the normalized estimated data model by using the estimated transfer function found from the minimization procedure $\left(\hat{\mathbf{S}}_{r}(\theta, f)\right)$.

The estimation of the transfer functions, for a given spatial frequency $k+k_{c}$, is assessed by minimizing the residual error between the measured data $\mathbf{S}_{r}(\theta, k)$ and the data model $\hat{\mathbf{S}}_{r}(\theta, k)$ such as the following [36]:
$\underset{\hat{h}(k)}{\operatorname{minimize}}\left|\hat{\mathbf{S}}_{r}(\theta, k)-\frac{1}{G(\theta, k)} \mathbf{S}_{r}(\theta, k)\right|^{2}$

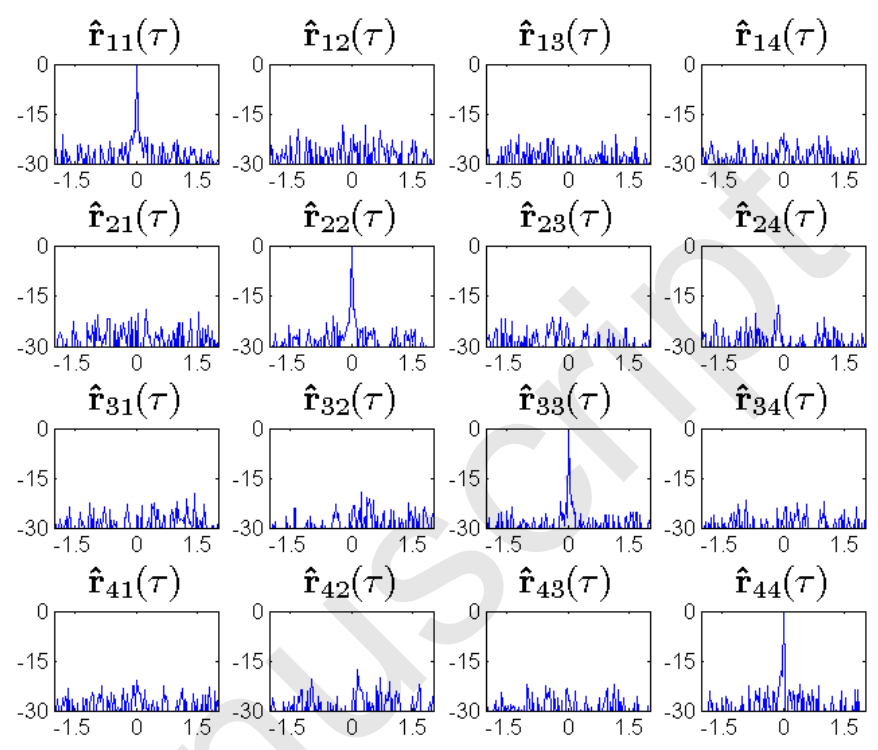

Fig. 12. $\hat{\mathbf{r}}_{\mathbf{i j}}(\tau)=D F T^{-1}\left[\hat{h}_{i}(f) \hat{h}_{j}(f)^{*}\right]$, the time domain normalized auto/cross-correlation results of the estimated transfer functions $(\hat{\mathbf{h}}(f))$, with $\tau$ [ns] along the horizontal axis and the normalized amplitude $[\mathrm{dB}]$ along the vertical axis.

The DUT is measured for $\theta \in\left[-90^{\circ}, 90^{\circ}\right]$ with $N_{\theta}=181$ scanning angles and $f+f_{c}=\left(k+k_{c}\right) c /(4 \pi) \in$ [50GHz, 66GHz] with $N_{f}=2001$. The corresponding measured data $\mathbf{S}_{r}(\theta, f)$ is shown in Fig. 10. The grating lobes, spatially filtered by the radiation pattern of each element, are present in the graph since the element spacing of $2.5 \mathrm{~cm}$ corresponds to $5 \lambda$ at $60 \mathrm{GHz}$.

The minimization is performed for a limited range of observation angles $\theta \in\left[-30^{\circ}, 30^{\circ}\right]$. Since isotropic radiating sources are used in the data model, the radiation pattern effects, in the measured data, are compensated prior to applying the minimization to estimate the transfer functions at each frequency (see Eq. 10)). Figs 11a and 11b compare the results of the measured data and the data model, at 50 $\mathrm{GHz}$, in amplitude and phase by using the estimated transfer function from the minimization. Fig. 12 shows the correlation between the estimated transfer functions from the plane-wave estimation method $(\hat{\mathbf{h}}(f))$. With regards to the correlation results, the PSLR of the correlation functions for each port (along the diagonal) is lower than $-17.5 \mathrm{~dB}$, with an ISLR lower than $-14.1 \mathrm{~dB}$. Regarding the cross-correlation results (above and below the diagonal), the highest peak reaches $-15 \mathrm{~dB}$. Fig. 13 shows the amplitude of the normalized complex inner product between the measured transfer vector $(\mathbf{h}(f))$ and the estimated transfer functions vector $(\hat{\mathbf{h}}(f))$. An inner product between two unitary vectors equals one if the two complex vectors are equals. The mean result along the frequency is 0.96 .

It is possible to estimate the transfer functions of a PCD whose ports are arranged in a uniform linear array according 


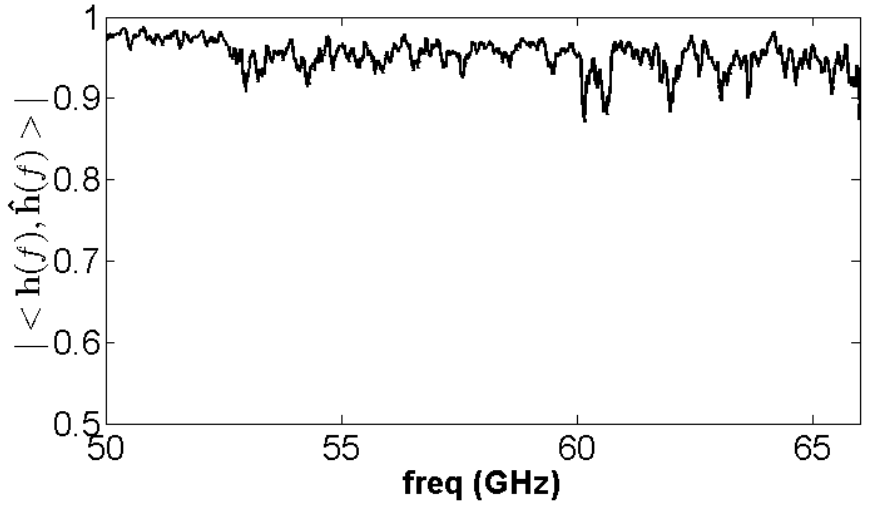

Fig. 13. Amplitude of the normalized inner product results between $\mathbf{h}(f)$ and $\hat{\mathbf{h}}(f)$, along the frequency.

to the plane-wave estimation method. With a non-uniform or sparse linear array, the method can also be applied. To reduce the number of acquisitions, it is possible to estimate the transfer function from sparse acquisitions when the spatial $k$-space is under-sampled by means of least squares minimization methods [37]. If the outputs of the PCD are located over a 2-Dimensional array, it is required to rotate along the azimuth and the elevation directions to extract the transfer functions in the same manner as in the 1-Dimensional case.

\section{MiMO CONFiguration MEASUREMEnts}

To validate the accuracy of the transfer function retrieval technique, measurements of the system point spread function are performed in a MIMO configuration. The configuration is shown in Fig. 14. The measurements are performed using the IETR facility DIADEM (DIagnostic, Analysis and Dosimetry of EM fields). This facility dedicated to electro-magnetic imaging is based on a $600 \times 600 \times 600 \mathrm{~mm}^{3}$ xyz scanner located in an anechoic chamber. The RF measurement system uses a classical architecture with a VNA and external VDI TxRef and Rx frequency extenders.

The four output ports of the PCD are used as transmitting antennas with element spacing, $\Delta_{x}$, equal to $25 \mathrm{~mm}$ with $\delta_{x}=$ $2.5 \mathrm{~mm}$, the element spacing of the receiving synthetic array. The receiving synthetic aperture is located over an aperture of length $\Delta_{x}-\delta_{x}$ and aligned with the x-axis. The location of each phase center is $\left(x_{a}, y_{a}, z_{a}\right)$ with $x_{a} \in\left[-\Delta_{x} / 2, \Delta_{x} / 2-\right.$ $\left.\delta_{x}\right]$. Following the same notation as in (4), the receiving signals along the continuous receiving aperture are expressed as:

$S_{r}\left(x_{a}, k\right)=\sum_{m=1}^{M} h_{m}(k) \cdot \sum_{i=1}^{N_{s}} s_{i} e^{-j\left(k+k_{c}\right) d_{i}\left(m, x_{a}\right)}+n\left(x_{a}, k\right)$

with

$$
\begin{aligned}
d_{i}\left(m, x_{a}\right) & =\left(d T x_{i}(m)+d R x_{i}\left(x_{a}\right)\right) / 2 \\
& =\left(\sqrt{\left(x_{t}(m)-x_{i}\right)^{2}+\left(y_{t}-y_{i}\right)^{2}+\left(z_{t}-z_{i}\right)^{2}}\right. \\
& \left.+\sqrt{\left(x_{a}-x_{i}\right)^{2}+\left(y_{a}-y_{i}\right)^{2}+\left(z_{a}-z_{i}\right)^{2}}\right) / 2
\end{aligned}
$$

with $\left\{x_{t}(m)=-\Delta_{x} \cdot(M-1) / 2+(m-1) \cdot \Delta_{x}-x_{0}\right\}_{m=1}^{M}$, and $x_{0}=20 \mathrm{~cm}$, a constant that corresponds to the distance

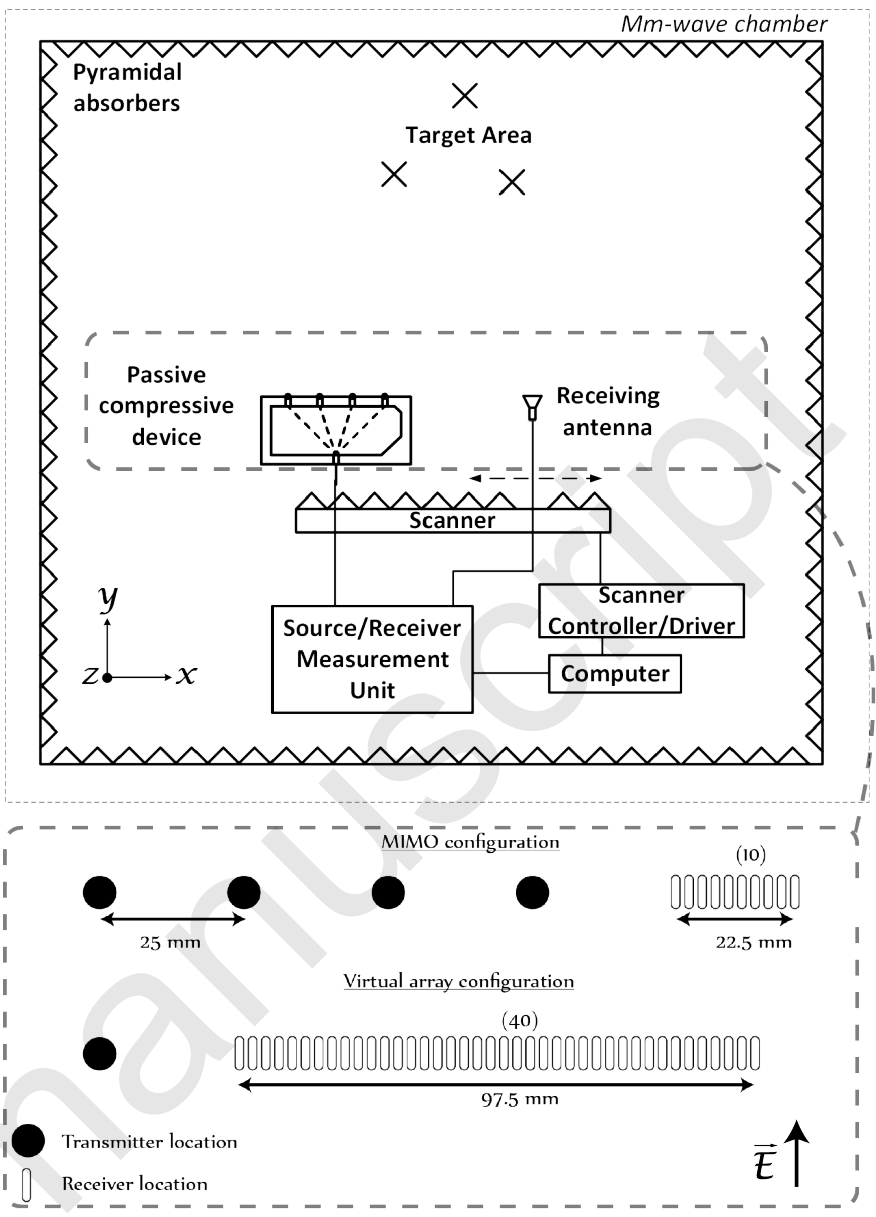

Fig. 14. Measurement setup.

between the center of the receiving aperture and the center of the PCD.

Similar to (5), four matched filters are applied to extract the signals received from the four output ports. $S_{m}\left(x_{a}, k\right)$ is the received signal, which comes from the $m^{\text {th }}$ output port on the receiving array $x_{a}$, after matched filtering. $Y_{m}\left(x_{a}, d\right)=$ $\operatorname{IFT}_{k}\left[S_{m}\left(x_{a}, k\right)\right]$ is the received signal in the spatial domain $d$.

Finally, the back-projection algorithm is used to generate the focused SAR image $f\left(x, y, z=C_{0}\right)$ with $C_{0}=0.3 \mathrm{~m}$ a constant height. The reflectivity at a particular location $p_{l}$ with Cartesian coordinate $\left(x_{l}, y_{l}, z_{l}\right)$ of the focused SAR image is constructed by:

$f\left(x_{l}, y_{l}, z_{l}\right)=\sum_{m=1}^{M} \int_{x_{a}=-\Delta_{x} / 2}^{\Delta_{x} / 2} Y_{m}\left(x_{a}, d_{l}\left(m, x_{a}\right)\right) e^{j k_{c} d_{l}\left(m, x_{a}\right)} \mathrm{d} x_{a}$

with $d_{l}\left(m, x_{a}\right)$ being the round-trip distance between the $m^{t h}$ port of the PCD, the $l^{\text {th }}$ pixel location and the receiving aperture $x_{a}$. The receiving array of length $\Delta_{x}-\delta_{x}=22.5$ $\mathrm{mm}$ is synthesized by moving the RF reception module to $\delta_{x}=2.5 \mathrm{~mm}$ spaced discrete positions with the scanner, which corresponds to an equivalent array of 10 elements. The MIMO configuration generates a virtual array with fewer elements. In terms of achievable cross-range resolution, it is comparable to 
one fixed transmitting element with 40 receiving elements. A $16 \mathrm{GHz}$ bandwidth signal $\left(B_{f}\right)$ with a $58 \mathrm{GHz}$ carrier frequency $\left(f_{c}\right)$ has been used. To speed up the acquisition of the 2001 frequency points obtained for each reception position, an IF filter of $100 \mathrm{KHz}$ was applied. It has to be noticed that even if the IF filter is broadband, the dynamic range provided by the VDI modules is high enough for the purposes of these tests.

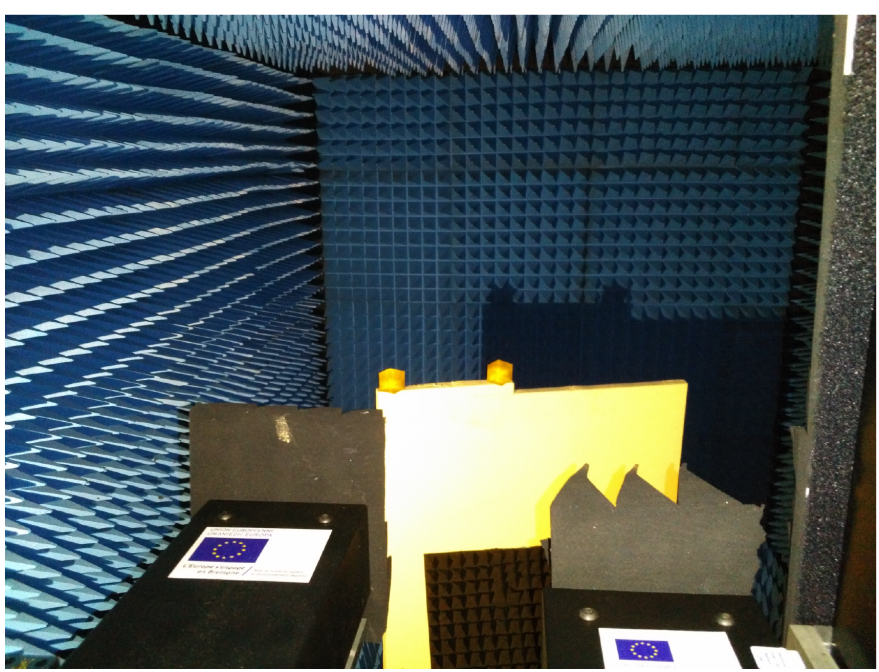

Fig. 15. Scene in the anechoic chamber composed of two corner reflectors.

Figure 15 shows two metallic trihedral corners with an edge length of $50 \mathrm{~mm}$ settled at about $1.4 \mathrm{~m}$ from the imaging system, on a foam support, whose relative electrical permittivity, close to one, minimize the sources of diffraction, and that are limited mainly to the backscattering of the anechoic chamber. This enables to measure the system point spread function. Fig. 16a shows the imaging result of the virtual array, synthesized by means of the scanner to perform a SIMO configuration as already shown in Fig. 14. The two target's positions can be retrieved. This result is used as a reference to be compared with the imaging results where the $\mathrm{PCD}$ is used with a smaller receiving array.

Figure 16b shows the imaging result of the MIMO configuration associated with the $\mathrm{PCD}$ in which the transfer functions are measured using the Port-to-Port measurements detailed in the Section III-A. Fig. 16c shows the imaging result of the MIMO configuration associated with the PCD in which the transfer functions are estimated with the plane-wave estimation method detailed in Section III-B. Table I summarizes and compares the point spread function performances in terms of range and cross-range resolutions and side lobe levels. When the transfer function has been estimated from the plane-wave estimation method, the range/cross-range resolution is closer to the performance of the reference (the virtual array) than when the transfer functions are measured from the Port-toPort measurement method.

\section{CONCLUSION}

A passive compressive device at millimeter-waves has been manufactured and used in a virtual array MIMO configuration

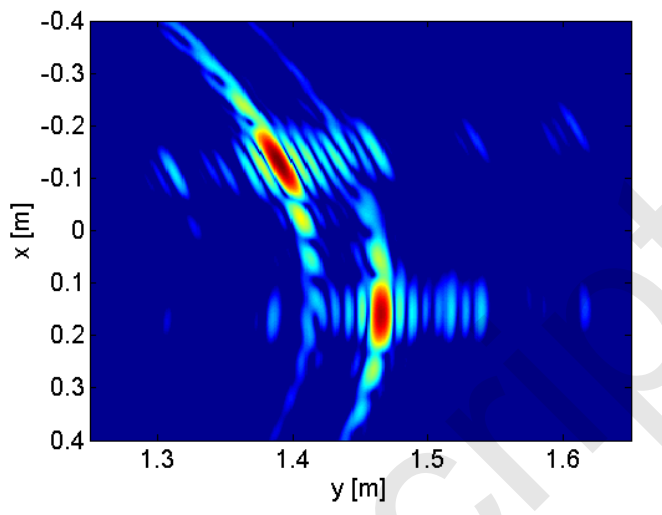

(a)

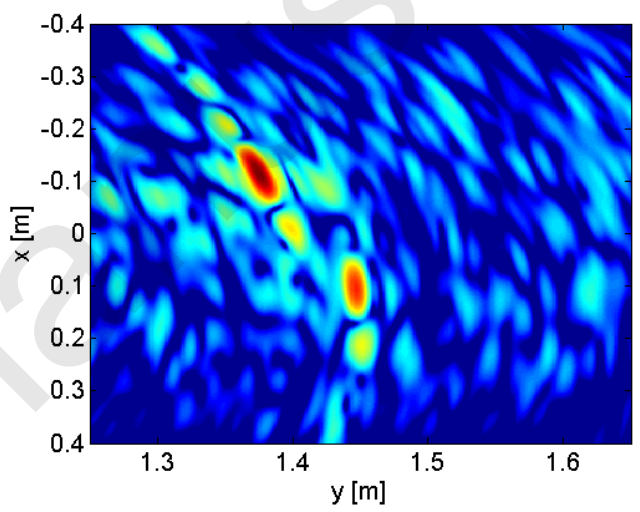

(b)

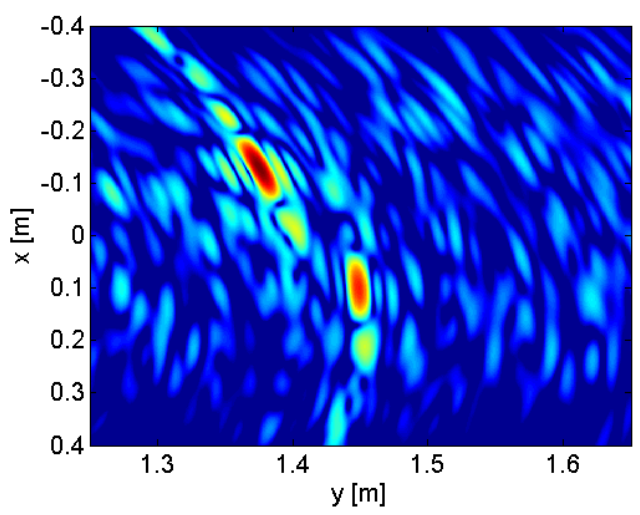

(c)

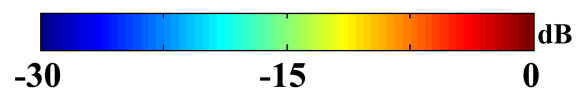

Fig. 16. Measurement imaging results using Eq. (13), of two isotropic targets (corner reflectors) captured by (a) a classic SIMO configuration with 1Tx (transmitting element) and 40 Rxs (receiving elements), (b) the PCD with 10 Rxs elements where the Port-to-Port measurement is used to have the transfer function, and (c) the PCD with 10 Rxs elements where the planewave estimation is used to have the transfer function. 
TABLE I

SUMMARIZED POINT SPREAD FUNCTION PERFORMANCES

\begin{tabular}{|l||l|l|l|}
\hline Performance & $\begin{array}{l}\text { Virtual ar- } \\
\text { ray }\end{array}$ & $\begin{array}{l}\text { Port-to-Port } \\
\text { measure- } \\
\text { ment }\end{array}$ & $\begin{array}{l}\text { plane-wave } \\
\text { estimation } \\
\text { method }\end{array}$ \\
\hline $\begin{array}{l}\text { Range resolution } \\
(\mathrm{mm})\end{array}$ & 10.4 & 11.8 & 10.6 \\
\hline $\begin{array}{l}\text { Cross-Range res- } \\
\text { olution (mm) }\end{array}$ & 61.7 & 62 & 61.7 \\
\hline $\begin{array}{l}\text { Side-lobe level } \\
(\mathrm{dB})\end{array}$ & -13.58 & -10.63 & -12.5 \\
\hline
\end{tabular}

and has shown to maintain a stable spatial resolution and low side lobe levels. Such a device is of considerable interest at millimeter-waves because, since the small wavelength permits manufacturing a compact component with significant decorrelation between the channels. It is a promising method of reducing the number of $\mathrm{Tx}$ and $\mathrm{Rx} \mathrm{RF}$ chains, hence the cost of radar imaging systems in numerous configurations and for many applications. Furthermore, the authors have shown a suitable method to estimate simultaneously the transfer functions of the passive compressive device with considerable accuracy. The estimated transfer functions have been used to image a static scene with two corner reflectors. Besides the simplicity of the proposed method, it outperforms classic portto-port transfer function measurements in terms of achievable spatial resolution and sidelobe level. The next step in this work concerns the realization of a switched receiving array based on wide-band stacked patch antennas to avoid the scanning process and to be used with the passive compressive device in an almost real-time MIMO radar imaging system.

\section{ACKNOWLEDGMENT}

The authors are grateful to Xavier Morvan for the mechanical set up and Laurent Le Coq for the help with measurements. This publication is supported by the European Union through the European Regional Development Fund (ERDF), and by Ministry of Higher Education and Research, Brittany and Rennes Metropole, through the CPER Project SOPHIE / STIC \& Ondes.

\section{REFERENCES}

[1] D. M. Sheen, D. L. McMakin, and T. E. Hall, "Three-dimensional millimeter-wave imaging for concealed weapon detection," IEEE Transactions on Microwave Theory and Techniques, vol. 49, no. 9, pp. 15811592, Sep 2001

[2] S. S. Ahmed, A. Schiessl, and L. P. Schmidt, "A Novel Fully Electronic Active Real-Time Imager Based on a Planar Multistatic Sparse Array," IEEE Transactions on Microwave Theory and Techniques, vol. 59, no. 12, pp. 3567-3576, Dec 2011.

[3] G. L. Charvat, L. C. Kempel, E. J. Rothwell, C. M. Coleman, and E. L. Mokole, "A Through-Dielectric Ultrawideband (UWB) SwitchedAntenna-Array Radar Imaging System," IEEE Transactions on Antennas and Propagation, vol. 60, no. 11, pp. 5495-5500, Nov 2012.

[4] X. Zhuge and A. G. Yarovoy, "Three-Dimensional Near-Field MIMO Array Imaging Using Range Migration Techniques," IEEE Transactions on Image Processing, vol. 21, no. 6, pp. 3026-3033, June 2012.

[5] J. Li and P. Stoica, "MIMO radar with colocated antennas," IEEE Signal Processing Magazine, vol. 24, no. 5, pp. 106-114, 2007.

[6] D.-w. Wang, X.-y. Ma, A.-L. Chen, and Y. Su, "High-resolution imaging using a wideband MIMO radar system with two distributed arrays," IEEE transactions on image processing, vol. 19, no. 5, pp. 1280-1289, 2010.
[7] D.-w. Wang, X.-y. Ma, and Y. Su, "Two-dimensional imaging via a narrowband MIMO radar system with two perpendicular linear arrays," IEEE Transactions on Image Processing, vol. 19, no. 5, pp. 1269-1279, 2010.

[8] D. W. Bliss and K. W. Forsythe, "Multiple-input multiple-output (MIMO) radar and imaging: degrees of freedom and resolution," in The Thrity-Seventh Asilomar Conference on Signals, Systems Computers, 2003, vol. 1, Nov 2003, pp. 54-59 Vol.1.

[9] G. Krieger, N. Gebert, and A. Moreira, "Multidimensional Waveform Encoding: A New Digital Beamforming Technique for Synthetic Aperture Radar Remote Sensing," IEEE Transactions on Geoscience and Remote Sensing, vol. 46, no. 1, pp. 31-46, Jan 2008.

[10] W. Q. Wang, "Time Coding MIMO-OFDM SAR for High-Resolution Imaging," IEEE Transactions on Geoscience and Remote Sensing, vol. 49, no. 8, pp. 3094-3104, Aug 2011.

[11] G. Krieger, "MIMO-SAR: Opportunities and Pitfalls," IEEE Transactions on Geoscience and Remote Sensing, vol. 52, no. 5, pp. 2628-2645, May 2014.

[12] A. D. Maio and M. Lops, "Design Principles of MIMO radar detectors," IEEE Transactions on Aerospace and Electronic Systems, vol. 43, no. 3, pp. 886-898, July 2007.

[13] J. Gollub, O. Yurduseven, K. Trofatter, D. Arnitz, M. Imani, T. Sleasman, M. Boyarsky, A. Rose, A. Pedross-Engel, H. Odabasi et al., "Large metasurface aperture for millimeter wave computational imaging at the human-scale," Scientific Reports, vol. 7, 2017.

[14] T. Sleasman, M. Boyarsky, M. F. Imani, J. N. Gollub, and D. R. Smith, "Design considerations for a dynamic metamaterial aperture for computational imaging at microwave frequencies," JOSA B, vol. 33, no. 6, pp. 1098-1111, 2016.

[15] G. Montaldo, D. Palacio, M. Tanter, and M. Fink, "Building threedimensional images using a time-reversal chaotic cavity," IEEE Transactions on Ultrasonics, Ferroelectrics, and Frequency Control, vol. 52, no. 9, pp. 1489-1497, Sept 2005

[16] O. Yurduseven, V. R. Gowda, J. N. Gollub, and D. R. Smith, "Printed Aperiodic Cavity for Computational and Microwave Imaging," IEEE Microwave and Wireless Components Letters, vol. 26, no. 5, pp. 367369, May 2016.

[17] M. Fink, "Time reversal of ultrasonic fields. i. Basic principles," IEEE Transactions on Ultrasonics, Ferroelectrics, and Frequency Control, vol. 39, no. 5, pp. 555-566, Sept 1992.

[18] A. Molaei, J. H. Juesas, and J. A. M. Lorenzo, "Compressive reflector antenna phased array," in Antenna Arrays and Beam-formation. InTech, 2017.

[19] O. Yurduseven, J. N. Gollub, K. P. Trofatter, D. L. Marks, A. Rose, and D. R. Smith, "Software Calibration of a Frequency-Diverse, Multistatic, Computational Imaging System," IEEE Access, vol. 4, pp. 2488-2497, 2016.

[20] D. Carsenat and C. Decroze, "UWB Antennas Beamforming Using Passive Time-Reversal Device," IEEE Antennas and Wireless Propagation Letters, vol. 11, pp. 779-782, 2012.

[21] T. Fromenteze, C. Decroze, and D. Carsenat, "Waveform Coding for Passive Multiplexing: Application to Microwave Imaging," IEEE Transactions on Antennas and Propagation, vol. 63, no. 2, pp. 593-600, Feb 2015.

[22] T. Fromenteze, D. Carsenat, and C. Decroze, "A precorrection method for passive UWB time-reversal beamformer," IEEE Antennas and Wireless Propagation Letters, vol. 12, pp. 836-840, 2013.

[23] R. Négrier, M. Lalande, J. Andrieu, B. M. Shalaby, V. Couderc, T. Fromenteze, C. Decroze, and D. Carsenat, "High-PRF UWB optoelectronic Radar system: a CLEAN-type algorithm to overcome depth limitation," IEEE Transactions on Antennas and Propagation, vol. 64, no. 3, pp. 1080-1088, 2016

[24] C. L. Liu and P. P. Vaidyanathan, "Super Nested Arrays: Linear Sparse Arrays With Reduced Mutual Coupling;Part I: Fundamentals," IEEE Transactions on Signal Processing, vol. 64, no. 15, pp. 3997-4012, Aug 2016.

[25] D. Tarchi, F. Oliveri, and P. F. Sammartino, "MIMO Radar and GroundBased SAR Imaging Systems: Equivalent Approaches for Remote Sensing," IEEE Transactions on Geoscience and Remote Sensing, vol. 51, no. 1, pp. 425-435, Jan 2013.

[26] A. Jouade, S. Meric, O. Lafond, M. Himdi, and L. F. Famil, "A passive compressive device associated with a luneburg lens for multi-beam radar at millimeter-wave," IEEE Antennas and Wireless Propagation Letters, pp. 1-1, 2018.

[27] A. Pedross-Engel, C. M. Watts, D. R. Smith, and M. S. Reynolds, "Enhanced Resolution Stripmap Mode Using Dynamic Metasurface 
Antennas," IEEE Transactions on Geoscience and Remote Sensing, 2017.

[28] D. Cerutti-Maori, I. Sikaneta, J. Klare, and C. H. Gierull, "MIMO SAR Processing for Multichannel High-Resolution Wide-Swath Radars," IEEE Transactions on Geoscience and Remote Sensing, vol. 52, no. 8, pp. 5034-5055, Aug 2014.

[29] P. Berardino, G. Fornaro, R. Lanari, and E. Sansosti, "A new algorithm for surface deformation monitoring based on small baseline differential SAR interferograms," IEEE Transactions on Geoscience and Remote Sensing, vol. 40, no. 11, pp. 2375-2383, 2002.

[30] M. Neumann, L. Ferro-Famil, and A. Reigber, "Multibaseline polarimetric SAR interferometry coherence optimization," IEEE Geoscience and Remote Sensing Letters, vol. 5, no. 1, pp. 93-97, 2008.

[31] L. Ferro-Famil, E. Pottier, and J.-S. Lee, "Unsupervised classification of multifrequency and fully polarimetric SAR images based on the H/A/Alpha-Wishart classifier," IEEE Transactions on Geoscience and Remote Sensing, vol. 39, no. 11, pp. 2332-2342, 2001.

[32] J.-B. Gros, O. Legrand, F. Mortessagne, E. Richalot, and K. Selemani, "Universal behaviour of a wave chaos based electromagnetic reverberation chamber," Wave Motion, vol. 51, no. 4, pp. 664-672, 2014.

[33] M. Soumekh, Synthetic aperture radar signal processing. New York: Wiley, 1999, vol. 7.

[34] X. Lu and H. Sun, "Parameter assessment for SAR image quality evaluation system," in 2007 1st Asian and Pacific Conference on Synthetic Aperture Radar, Nov 2007, pp. 58-60.

[35] L. Le Coq, B. Fuchs, T. Kozan, S. Burgos, and P. O. Iversen, "IETR millimeter-wave Compact Antenna Test Range implementation and validation," in 2015 9th European Conference on Antennas and Propagation $($ EuCAP). IEEE, 2015, pp. 1-5.

[36] D. W. Marquardt, "An algorithm for least-squares estimation of nonlinear parameters," Journal of the society for Industrial and Applied Mathematics, vol. 11, no. 2, pp. 431-441, 1963.

[37] E. J. Candes, J. Romberg, and T. Tao, "Robust uncertainty principles: exact signal reconstruction from highly incomplete frequency information," IEEE Transactions on Information Theory, vol. 52, no. 2, pp. 489-509, Feb 2006

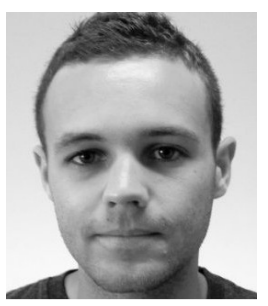

Antoine Jouadé received the M.Sc. degree in signal processing and telecommunication engineering from the University of Rennes1, Rennes, France and the Ph.D. degree in signal processing and telecommunications with the Institute of Electronics and Telecommunications of Rennes, University of Rennes I in 2017. His research interest is on imaging RADAR and associated antennas at millimeterwaves. He was an intern with the Antenna and Sub-millimeter Section of the European Space and Technology Center of the European Space Agency, ESA ESTEC, Noordwijk, The Netherlands and is currently working as a research engineer at the French Aerospace Laboratory (ONERA) in the Electromagnetism and Radar Department.

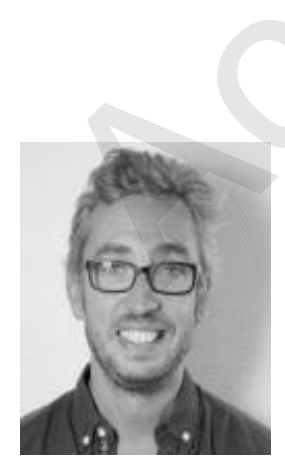

Olivier Lafond received his M.S. degree in Radar and Telecommunications from the University of Rennes 1, Rennes, France, in 1996, and the Ph.D. degree in Signal Processing and Telecommunications from the University of Rennes 1, Rennes, France, in 2000. Since October 2002, he has been an Associate Professor with the Institute of Electronics and Telecommunications of Rennes (IETR), University of Rennes 1 . He has authored or co-authored more than 30 journal papers and 50 papers in conference proceedings. He has also authored/co-authored three book chapters. He holds six patents in the area of antennas. His research activities deal with passive and active millimeter-waves multilayer antennas and circuits, reconfigurable antennas, inhomogeneous lenses for shaping radiation patterns with active devices, and imaging antenna systems.

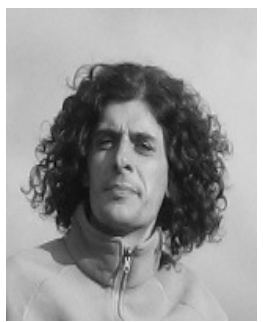

Laurent Ferro-Famil received the M.S. degree in electronic systems and computer engineering and the Ph.D. degree from the University of Nantes, Nantes, France, in 1996 and 2000, respectively. In 2001, he became an Associate Professor with the University of Rennes 1, Rennes, France. Since 2011, he has been a Full Professor with the University of Rennes 1 , where he is currently the Head of the Remote Sensing Department, Institute of Electronics and Telecommunications of Rennes. His current research interests include analog electronics, digital communications, microwave theory, signal processing, and polarimetric SAR remote sensing, polarimetric SAR signal statistical processing, radar polarimetry theory, and natural media remote sensing using multibaseline PolInSAR data, with application to classification, electromagnetic scattering modeling and physical parameter retrieval, time-frequency analysis, and 3-D reconstruction of environments using SAR tomography.

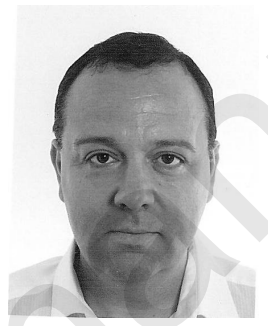

Mohamed Himdi received the Ph.D. degree in signal processing and telecommunications from the University of Rennes 1, Rennes, France, in 1990 Since 2003, he has been a Professor with the University of Rennes 1, and the Head of the High Frequency and Antenna Department until 2013, Institut dElectronique et Telecommunications de Rennes (IETR), Unite Mixte de Recherche, Center National de la Recherche Scientifique. He has authored or coauthored 90 journal papers and over 220 papers in conference proceedings. He has also authored/ coauthored six book chapters. He holds 33 patents in the area of antennas. His research activities concern passive and active millimeter-waves antennas. His research interests also include theoretical and applied computational electromagnetics, development of new architectures of printed antenna arrays, and new three-dimensional (3-D) antenna technologies. Pr. Himdi was the recipient of the 1992 International Symposium on Antennas and Propagation (ISAP) Conference Young Researcher Scientist Fellowship (Japan) and a 1995 Award presented by the International Union of Radio Scientists (Russia). He was Laureat of the Second National Competition for the Creation of Enterprises in Innovative Technologies in 2000 (Ministry of Industry and Education, France). In March 2015 he received the JECAWARD- 10 at Paris on Pure composite material antenna embedded into a motorhome roof for the Digital Terrestrial Television reception.

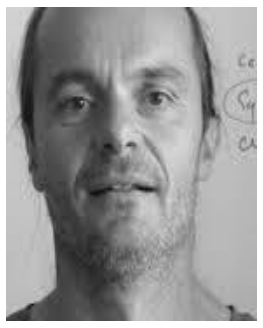

Stéphane Méric received the Diploma in electrical engineering from the National Institute for the Applied Sciences (INSA), Rennes, France, and the M.S. degree in signal processing and telecommunications from the University of Rennes 1, Rennes, France, both in 1991, and the Ph.D. degree in electronics from INSA in 1996. Since 2000, he has been an Assistant Professor with INSA, and in 2005, he joined the SAPHIR team (IETR CNRS UMR 6164, Rennes). His research interests include using SAR data in radargrammetric applications. Furthermore, he is currently working on radar system $(\mathrm{CW}, \mathrm{FMCW})$ dedicated to specific SAR applications (radar imaging in motorway context, remote sensing, MIMO configuration, passive radar imaging). His education activities are on analog electronics, signal processing, radar and radar imaging, and electromagnetic diffraction. 